\title{
Plant defensin antibacterial mode of action against Pseudomonas species
}

\author{
Andrew E. Sathoff ${ }^{1,2^{*}}$ (D), Shawn Lewenza ${ }^{3,4}$ and Deborah A. Samac ${ }^{1,5}$
}

\begin{abstract}
Background: Though many plant defensins exhibit antibacterial activity, little is known about their antibacterial mode of action (MOA). Antimicrobial peptides with a characterized MOA induce the expression of multiple bacterial outer membrane modifications, which are required for resistance to these membrane-targeting peptides. Mini-Tn5lux mutant strains of Pseudomonas aeruginosa with Tn insertions disrupting outer membrane protective modifications were assessed for sensitivity against plant defensin peptides. These transcriptional lux reporter strains were also evaluated for lux gene expression in response to sublethal plant defensin exposure. Also, a plant pathogen, Pseudomonas syringae pv. syringae was modified through transposon mutagenesis to create mutants that are resistant to in vitro MtDef4 treatments.

Results: Plant defensins displayed specific and potent antibacterial activity against strains of $P$. aeruginosa. A defensin from Medicago truncatula, MtDef4, induced dose-dependent gene expression of the aminoarabinose modification of LPS and surface polycation spermidine production operons. The ability for MtDef4 to damage bacterial outer membranes was also verified visually through fluorescent microscopy. Another defensin from M. truncatula, MtDef5, failed to induce lux gene expression and limited outer membrane damage was detected with fluorescent microscopy. The transposon insertion site on MtDef4 resistant $P$. syringae pv. syringae mutants was sequenced, and modifications of ribosomal genes were identified to contribute to enhanced resistance to plant defensin treatments.

Conclusions: MtDef4 damages the outer membrane similar to polymyxin B, which stimulates antimicrobial peptide resistance mechanisms to plant defensins. MtDef5, appears to have a different antibacterial MOA. Additionally, the MtDef4 antibacterial mode of action may also involve inhibition of translation.
\end{abstract}

Keywords: Plant defensin, Antibacterial, Medicago, Pseudomonas, lux reporter

\section{Background}

Plants produce a suite of antimicrobial peptides (AMPs) to defend against the extensive array of potential pathogens encountered in their environment. Plant AMPs are classified based on their structure and presence of disulfide bonds [1]. With an abundance of representatives from diverse plant species, plant defensins are among the most

\footnotetext{
* Correspondence: Andrew.Sathoff@dsu.edu

'Department of Plant Pathology, 1991 Upper Buford Circle, University of Minnesota, St. Paul, MN 55108, USA

${ }^{2}$ Department of Biology, Dakota State University, 820 N Washington Ave, Madison, SD 57042, USA

Full list of author information is available at the end of the article
}

widespread and best characterized plant AMPs [2]. Plant defensins are cationic, cysteine-rich antimicrobial peptides that usually contain four disulfide bonds. They have a conserved three-dimensional structure, a cysteine-stabilized $a \beta(\mathrm{CS} a \beta)$ motif, with a concentration of positively charged amino acid residues on the $\beta 2$ - $\beta 3$ loop, which is classified as the $\gamma$-core motif $\left(\mathrm{GXCX}_{3-9} \mathrm{C}\right)$. The $\gamma$-core motif alone has been shown to impart antimicrobial activity and mimic the activity of the corresponding full-length defensin [3]. Plant defensins are promiscuous peptides, which means that a single peptide can have multiple distinct functions [4]. Along with having antimicrobial activity, plant defensins control plant development, contribute

(c) The Author(s). 2020 Open Access This article is licensed under a Creative Commons Attribution 4.0 International License, which permits use, sharing, adaptation, distribution and reproduction in any medium or format, as long as you give appropriate credit to the original author(s) and the source, provide a link to the Creative Commons licence, and indicate if changes were made. The images or other third party material in this article are included in the article's Creative Commons licence, unless indicated otherwise in a credit line to the material. If material is not included in the article's Creative Commons licence and your intended use is not permitted by statutory regulation or exceeds the permitted use, you will need to obtain permission directly from the copyright holder. To view a copy of this licence, visit http://creativecommons.org/licenses/by/4.0/. The Creative Commons Public Domain Dedication waiver (http://creativecommons.org/publicdomain/zero/1.0/) applies to the data made available in this article, unless otherwise stated in a credit line to the data. 
to zinc tolerance, and act as inhibitors of digestive enzymes [5]. In crop plants, the transgenic expression of plant defensins has been used to engineer fungal and oomycete disease resistant plants. When MsDef1, a defensin from alfalfa (Medicago sativa), was expressed in potato, field-grown potatoes displayed resistance to Verticillium dahliae [6]. NaD1, a defensin from sweet tobacco (Nicotiana alata), provided transgenic cotton with resistance to Fusarium oxysporum f. sp. vasinfectum and $V$. dahliae throughout 3 years of field trials [7].

Though considered to be primarily antifungal, plant defensins have been shown to demonstrate antibacterial activity against both plant and vertebrate bacterial pathogens [8]. Spinach defensin (So-D2) is the most frequently cited plant defensin with antibacterial activity, and transgenic sweet orange and grapefruit trees expressing So-D2 exhibited increased resistance to the bacterial diseases, citrus greening and citrus canker, caused by Candidatus Liberibacter spp. and Xanthomonas axonopodis pv. citri, respectively [9]. Plant defensins also display in vitro antibacterial activity against human pathogens. For instance, J1-1, a defensin from bell pepper (Capsicum annum) has a minimum inhibitory concentration (MIC) value of $250 \mu \mathrm{g} / \mathrm{mL}$ against Pseudomonas aeruginosa [10]. Also, PaDef, a defensin from avocado (Persea americana var. drymifolia), displays antibacterial activity against Staphylococcus aureus [11]. Therefore, plant defensins not only appear to be a resource for improving plant immunity to bacterial diseases but also for combatting human and animal bacterial pathogens.

A major obstacle blocking the widespread usage of plant defensins as antibacterial compounds is that their antibacterial mode of action (MOA) is poorly characterized [8] although their MOA against fungal pathogens is well-described [12-14]. Recently, the antibacterial activity of a defensin from Medicago truncatula, MtDef5, was characterized [15]. MtDef5 is a bi-domain defensin with two defensin domains (MtDef5A and MtDef5B) connected by a 7-amino acid linker peptide. The cationic amino acid residues found in both $\gamma$-core motifs of MtDef5 were mutated and discovered to be essential for antibacterial activity, which were the same residues previously found to be essential for antifungal activity [16]. Additionally, MtDef5 was shown to permeabilize the plasma membrane of Xanthomonas campestris pv. campestris, a gram-negative bacterial plant pathogen, but not the gram-positive plant pathogen Clavibacter michiganensis subsp. nebraskensis [15]. The MtDef5 peptide binds to DNA indicating that it may kill bacterial cells by inhibiting DNA synthesis or transcription.

The MOA of human and invertebrate defensins against bacterial pathogens is well characterized $[17,18]$. Vertebrate defensins interact with the negatively charged lipopolysaccharide (LPS) in the bacterial outer membrane, which leads to swift permeabilization through pore formation [19]. For instance, HNP-1, the most investigated human $\alpha$-defensin, has an antibacterial MOA typical of many AMPs. HNP-1 dimerization occurs, and through electrostatic interactions of dimers with the bacterial membrane, $\beta$ sheet dimers cross the membrane forming a pore with higher order oligomers of HNP-1 forming upon the dimers when HNP-1 is in high concentration [20]. Human $\beta$ defensin-3 (HBD3) has another well-studied antibacterial MOA. HBD3 inhibits bacterial cell wall biosynthesis through interactions with lipid II components, which enables HBD3 to have broad-spectrum antibacterial activity against both gram-positive and gram-negative bacterial species [21].

In response to the electrostatic interactions between cationic AMPs and negatively charged bacterial membranes, gram-positive and gram-negative bacteria have demonstrated the ability to modify their membrane surfaces [22]. In $P$. aeruginosa and many other gram-negative bacteria, the PhoPQ/PmrAB systems control various genes required for resistance to AMPs [23]. The $p m r$ operon (PA3552-PA3559) is controlled by both PhoPQ and $\mathrm{PmrAB}$ and is required for the addition of aminoarabinose to mask the phosphates of lipid A in $P$. aeruginosa [24]. Upstream of $P m r A B$, the spermidine synthesis genes PA4773 (speD2) and PA4774 (speE2) in P. aeruginosa are required for production of this polycation on the outer surface of the bacterial membrane [25]. These surface modifications protect bacteria from cationic AMPs through masking of the negative surface charges, which limits AMP binding to bacterial membranes [24, 25]. The mini-Tn5-luxCDABE mutant library in $P$. aeruginosa has been used extensively to identify antimicrobial peptide MOAs and bacterial resistance mechanisms [26].

Pseudomonas syringae pv. syringae is a bacterial plant pathogen that causes bacterial stem blight of alfalfa, which is an economically important disease with widespread distribution in the Western United States [27]. Currently, there are no effective means to control bacterial stem blight of alfalfa. P. syringae pv. syringae strain ALF3, pathogenic on alfalfa and M. truncatula, has a draft genome sequence [28] and was shown to be sensitive to $M$. truncatula defensins, MtDef5 and MtDef4, with $\mathrm{IC}_{50}$ values of 0.1 and $0.4 \mu \mathrm{M}$, respectively [3]. Additionally, MtDef4 displays activity against Xanthomonas alfalfae subsp. alfalfae and the gram-positive bacterium Clavibacter insidiosus, while MtDef5 displays no activity against these pathogens [3]. There is insufficient knowledge to explain this observed specificity of plant defensin antibacterial activity.

In this study, we investigated plant defensin MOA against plant and vertebrate bacterial pathogens belonging to the genus Pseudomonas. Characterized $P$. aeruginosa lux-reporter strains with mutations in genes involved with cationic antimicrobial peptide resistance mechanisms 
were screened for sensitivity to $\gamma$-core motif plant defensin peptides. Transposon insertion libraries of $P$. syringae pv. syringae were generated and screened for plant defensin resistance. Generating tools to explore plant defensin MOA against bacterial plant pathogens is necessary for evaluating the risk of bacterial evolution towards defensin resistance and for the development of plant defensins into a spray-on peptide-based biological pesticide or transgenic expression of defensins for plant protection. Furthermore, knowing the antibacterial MOA of plant defensins will enhance their usage as antibacterial compounds and allow for prediction of antibacterial activity without extensive in vitro testing.

\section{Results}

\section{Plant defensin derived inhibition of Pseudomonas} aeruginosa growth

The antibacterial activity of $\gamma$-core motif peptides from MtDef4, MtDef5A, and So-D2 (Table 1) were evaluated against wild-type and antimicrobial peptide sensitive mutants of $P$. aeruginosa (Table 2). The $P$. aeruginosa lux $x$-reporter strains had inactivated LPS modification genes, either an interrupted outer membrane surface spermidine synthesis gene (PA4774) or an interrupted lipid A aminoarabinose modification gene (PA3553). These mutants are incapable of producing outer membrane surface modifications used for protection against cationic antimicrobial peptide treatments [26, 30,31]. Using a spread-plate assay, the $y$-core motif peptides exhibited antibacterial activity at micromolar concentrations. Against P. aeruginosa PAO1, the $y$-core peptides inhibited bacterial growth with MtDef4 displaying the greatest activity corresponding to an $\mathrm{IC}_{50}$ value of $4.2 \mu \mathrm{M}$ (Table 3). The lux-reporter $P$. aeruginosa strains had the expected increase in sensitivity towards both MtDef4 and So-D2 peptides compared to the wild type strain (Table 3). Overall, MtDef5 displayed the least antibacterial activity of the evaluated $y$-core motif defensin peptides with the highest recorded $\mathrm{IC}_{50}$ value of $14.6 \mu \mathrm{M}$ against PA4774::lux. In contrast, MtDef4 was the most potent against $P A 4774:: l u x$ with an $\mathrm{IC}_{50}$ value of $1.7 \mu \mathrm{M}$.

\section{Antimicrobial peptide resistance operons are induced by the MtDef4 $\gamma$-core motif peptide}

Transcriptional lux reporters of the $P$. aeruginosa $p m r$ operon (PA3552-PA3559) and spermidine synthesis genes speD2E2 (PA4773-PA4774) have been previously

Table 1 Amino acid sequences of $\gamma$-core motif (bold) and Cterminal region (italics) of plant defensins tested in vitro

\begin{tabular}{lll}
\hline Plant Species & Defensin & Amino Acid Sequence \\
\hline Medicago truncatula & MtDef4 & GRCRGFRRRCFCTTHC \\
M. truncatula & MtDef5A & GACHRQGFGFACFCYKKC \\
Spinacia oleracea & So-D2 & GDCKGIRRRCMCSKPL \\
\hline
\end{tabular}

shown to be induced by a $\mathrm{Mg}^{2+}$ limiting environment, acidic $\mathrm{pH}$, the presence of extracellular DNA, or the presence of antimicrobial peptides at a sublethal concentration [23, 30, 31]. We used these lux reporters under non-inducing conditions (diluted LB) to determine if exposure of these reporters to sub-MIC concentrations of plant defensins causes induced expression. The lux-reporter strains of $P$. aeruginosa were grown overnight in diluted LB broth, treated with plant defensin $\gamma$-core motif peptides, and monitored for bioluminescence in a microplate reader, where bioluminescence would indicate the induction of the inactivated bacterial membrane modification genes. Therefore, if the $\gamma$-core peptides cause bacterial membrane stress, the lux-reporter will be induced and bioluminescence will be recorded. In response to MtDef4 treatment at sublethal concentrations, lux expression was greatly induced in a concentration dependent manner in PA4774:: lux compared to PA3553:: lux (Fig. 1). For PA4774::lux, the level of induction from a treatment of $30 \mu \mathrm{g} / \mathrm{mL}$ of MtDef4 was greater than the induction caused by the antibiotic positive control, polymyxin B $(0.5 \mu \mathrm{g} / \mathrm{mL})$. Additionally, PA3553::lux expression was induced by MtDef4 at levels near those achieved by polymyxin B. MtDef5 and So-D2 failed to induce lux expression at levels near or greater than the antibiotic control in all mutant strains evaluated (Fig. 2). However, during the first $3 \mathrm{~h}$ after defensin treatment, the level of induced lux expression caused by all plant defensin treatments was greater than the antibiotic control, which indicates different kinetics and possibly MOAs between plant defensins and polymyxin B (Figs. 1 and 2).

\section{LIVE/DEAD BacLight staining of defensin treated Pseudomonas aeruginosa}

The LIVE/DEAD BacLight kit consists of two fluorescent nucleic acid stains: green-fluorescent SYTO 9 stain and red-fluorescent propidium iodine stain. SYTO 9 labels bacterial cells with both intact and damaged membranes, but propidium iodine can only penetrate and label bacteria with damaged membranes. Therefore, bacteria with intact membranes will fluoresce green, while bacteria with damaged membranes will fluoresce red [32]. Both PA4774::Iux and PA3553::lux were treated with either the MtDef4 or MtDef5 $\gamma$-core motif peptide and were stained with the BacLight kit. MtDef4 appeared to damage the bacterial membranes of both strains, especially PA4774::lux (Table 4, Fig. S1). MtDef5 seemed to cause limited bacterial outer membrane damage compared to MtDef4 (Table 4, Fig. S2).

\section{Pseudomonas syringae pv. syringae transposon mutagenesis}

The prior application of the mini-Tn5-luxCDABE mutant library in $P$. aeruginosa lead us to develop transposon- 
Table 2 Bacterial strains used in this study

\begin{tabular}{|c|c|c|}
\hline $\begin{array}{l}\text { Strain or } \\
\text { Mutant }\end{array}$ & Description & Reference \\
\hline PAO1 & Wild-type Pseudomonas aeruginosa & [29] \\
\hline PA3553::/ux & $\begin{array}{l}\text { Transposon mutants and transcriptional fusion, homolog to } p m r \text { gene ( } p m r F) \text { responsible for the addition of } \\
\text { aminoarabinose to lipid A }\end{array}$ & [26] \\
\hline PA4774::/UX & Transposon mutant and transcriptional fusion, homolog to speE gene responsible for spermidine synthesis & [26] \\
\hline ALF3 & Wild-type Pseudomonas syringae pv. syringae & [28] \\
\hline ALF3::Tn5-1 & ALF3 with random transposon insertion conferring MtDef4 insensitivity, Mu_4-1 & $\begin{array}{l}\text { This } \\
\text { paper }\end{array}$ \\
\hline ALF3::Tn5-2 & ALF3 with random transposon insertion conferring MtDef4 insensitivity, Mu_5-1 & $\begin{array}{l}\text { This } \\
\text { paper }\end{array}$ \\
\hline
\end{tabular}

insertion mutant screen of a bacterial plant pathogen, $P$. syringae pv. syringae strain ALF3. The $\gamma$-core motif peptide of MtDef4 was previously shown to have an $\mathrm{IC}_{50}$ value of $3.4 \mu \mathrm{M}$ against $P$. syringae pv. syringae [3], so the transposon-insertion mutants were screened for resistance at $40 \mu \mathrm{M}$ MtDef4 $\left(10 \times \mathrm{IC}_{50}\right)$. Out of thousands of plated colonies, three slow-growing, MtDef4 resistant $P$. syringae pv. syringae mutants were recovered following two repetitions of the $\gamma$-core defensin peptide treatment. Genomic DNA was extracted, digested with EcoRI, and self-ligated with T4 DNA ligase to generate plasmids that were transformed into E. coli. Plasmid DNA surrounding the transposon insertion sites was sequenced for two mutants. Although sequencing was attempted from several clones of the third mutant, no sequence was obtained suggesting that the mutation was not due to a transposon insertion. The resulting sequence data from the two MtDef4 insensitive mutants (ALF3::Tn5-1 and ALF3::Tn5-2) were characterized using BLAST analyses. The transposon insertion sites for both ALF3::Tn5-1 and ALF3::Tn5-2 were found to be located on scaffold 32544_1.1 of the ALF3 genome assembly and are 3824 base pairs apart. The mutated gene (RS24240) in ALF3::Tn5-1 is annotated as a $16 \mathrm{~S}$ ribosomal RNA gene, and the mutated gene (RS24220) in ALF3:: $\operatorname{Tn} 5-2$ is annotated as a $23 \mathrm{~S}$ ribosomal RNA gene (Table 5).

\section{Discussion}

Plant defensins are able to kill a broad spectrum of gram-positive and gram-negative bacteria, and therefore,

Table 3 Activity of the $y$-core motif defensin peptides against Pseudomonas aeruginosa strains ${ }^{a}$

\begin{tabular}{llll}
\hline Pseudomonas aeruginosa strains & $\begin{array}{l}\text { MtDef4 } \\
\text { core }\end{array}$ & $\begin{array}{l}\text { MtDef5A } \\
\text { core }\end{array}$ & $\begin{array}{l}\text { So-D2 } \\
\text { core }\end{array}$ \\
\hline PAO1 & $4.2 \pm 0.4$ & $11.8 \pm 1.4$ & $11.6 \pm 0.6$ \\
PA3553:Iux & $2.7 \pm 0.3$ & $8.5 \pm 0.8$ & $3.0 \pm 0.3$ \\
PA4774:Iux & $1.7 \pm 0.2$ & $14.6 \pm 1.0$ & $5.2 \pm 0.5$ \\
\hline
\end{tabular}

${ }^{a}$ The mean $\mathrm{IC}_{50}(\mu \mathrm{M})$ values are reported $\pm \mathrm{SE}$ of three independent experiments $(n=3)$ they are valuable candidates for generating a new class of antibiotics to treat multidrug-resistant bacteria. Fulllength defensin peptides have $\mathrm{IC}_{50}$ values approximately ten-fold lower than those from the corresponding $\gamma$-core motif peptides [3], which indicates that full-length defensins may have a more nuanced MOA where another undiscovered motif may be acting in synergy with the $\gamma$ core. A major drawback of peptide-based antibiotics is that they are much more expensive to produce than conventional antibiotics, so to reduce cost, the size of the peptide should be minimized [33]. Truncated plant defensins ( $\gamma$-core motif peptides) were assessed to evaluate a more realistic peptide-based antibacterial treatment. The plant defensin $\gamma$-core motif peptides demonstrated potent activity against $P$. aeruginosa (Table 3).

Gram-negative bacteria contain an outer membrane composed of LPS in the outer leaflet. Divalent inorganic cations $\left(\mathrm{Mg}^{2+}\right.$ and $\left.\mathrm{Ca}^{2+}\right)$ stabilize the outer leaflet by binding neighboring LPS molecules, and the displacement of these cations by antimicrobial peptides results in membrane destabilization and bacterial cell death [34]. Polycation spermidine production and aminoarabinosemodification of lipid A contribute to reduce outer membrane permeability and therefore, the entrance of cationic AMPs [25, 35]. Random mini-Tn5 transposon mutagenesis has been performed on P. aeruginosa PAO1, and the sites flanking the insertion have been sequenced and mapped, which has allowed for the characterization of outer membrane modification mutants [26]. These P. aeruginosa membrane modification mutants have increased sensitivity to MtDef4 and So-D2 $y$-core motif peptides with $\mathrm{IC}_{50}$ values reduced by $2-4$ fold compared to PAO1 (Table 3). This suggests that these plant defensins may have a MOA that involves pore creation in which the displacement of divalent metal cations causes destabilization of the LPS and disruption of membrane integrity. When evaluated against the MtDef5A $y$-core motif peptide, PA3553::lux shows a limited increase in susceptibility and $P A 4774:: l u x$ has a modest increased resistance. This lack of greatly enhanced susceptibility implies that MtDef5 
a

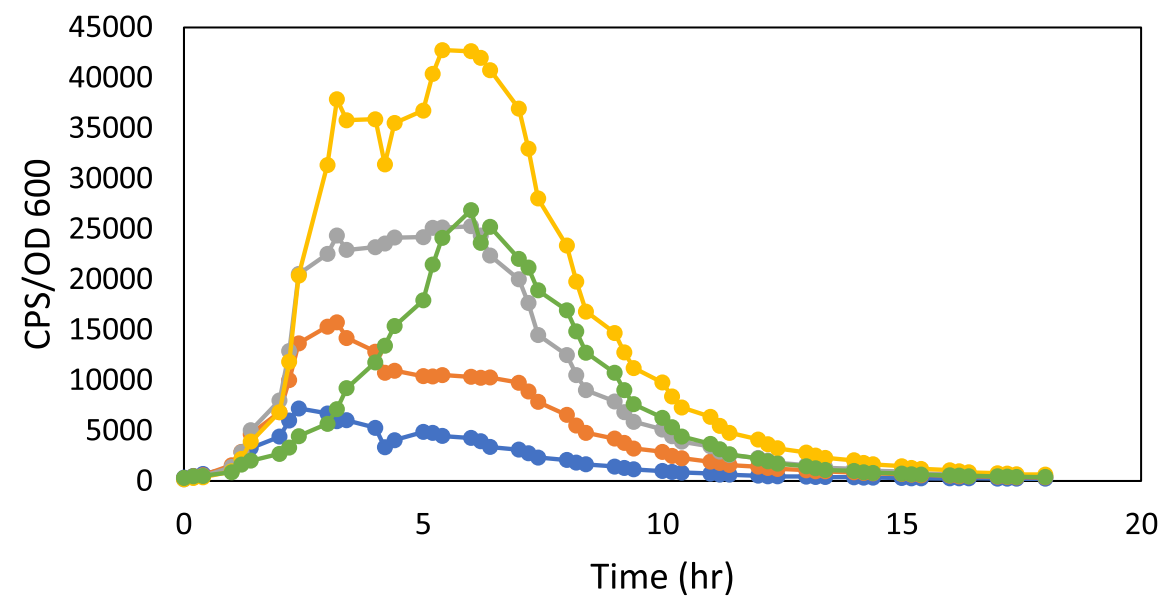

$\multimap 0 \multimap-5 \multimap-15 \multimap-30 \multimap-$ Polymyxin B

b

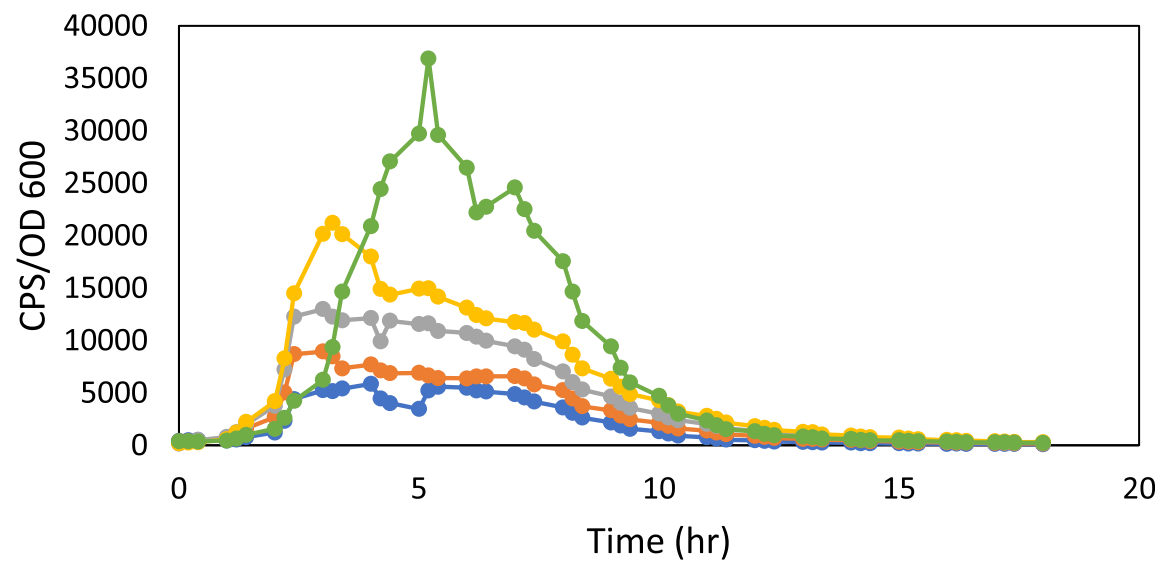

$\multimap 0 \multimap-5 \multimap-15 \multimap-30 \multimap-$ Polymyxin B

Fig. 1 MtDef4 $\gamma$-core motif peptide induces PA4774 and early PA3553 gene expression. Effects of MtDef4 $\gamma$-core peptide at sub-minimal inhibitory concentrations of $0,5,15$, or $30 \mu \mathrm{g} / \mathrm{mL}$ or polymyxin B at $0.5 \mu \mathrm{g} / \mathrm{mL}$ on the expression of the PA4774::/ux (a) and PA3553:./ux (b) transcriptional fusion in planktonic cultures in LB broth. Gene expression was normalized for growth and counts per second (CPS)/OD600 values for the average of the triplicates are presented. Each growth experiment was performed three times and representative curves are shown. The standard errors were within $10 \%$ of the mean

does not directly act on the bacterial outer membrane and may have an intracellular target considering that MtDef5 does not induce gene expression of the reporters. Accordingly, the fluorescent microscopy images revealed that MtDef5 caused limited outer membrane damage (Fig. S2). MtDef5 was previously shown to be internalized in $X$. campestris pv. campestris [15]. Also, MtDef5 demonstrates no activity towards gram-positive pathogens, $C$. insidiosus and $C$. nebraskensis, while MtDef4 had high antibacterial activity against $C$. insidiosus $[3,15]$. This could be due to the inability of MtDef5 to enter the bacterial cell through the thick outer layer of peptidoglycan present in the cell wall of gram-positive cells and interact with its intracellular target. These results suggest differing MOAs between MtDef5 and the other plant defensins evaluated.

The $P$. aeruginosa mini-Tn5-luxCDABE mutants contain a promoterless luciferase gene cassette, which serves as a sensitive, real-time reporter of gene expression for the inactivated gene [26]. Highly induced expression of the lux gene following plant defensin treatments at sublethal concentrations signals that the defensin peptide acts on the bacterial membrane, similar to other known antimicrobial peptides [23]. MtDef4 $y$-core motif peptide treatments cause a strong concentration-dependent induction of lux in the $P$. aeruginosa mutant, PA4774::lux (Fig. 1). PA4774::lux also displayed the greatest level of 
a

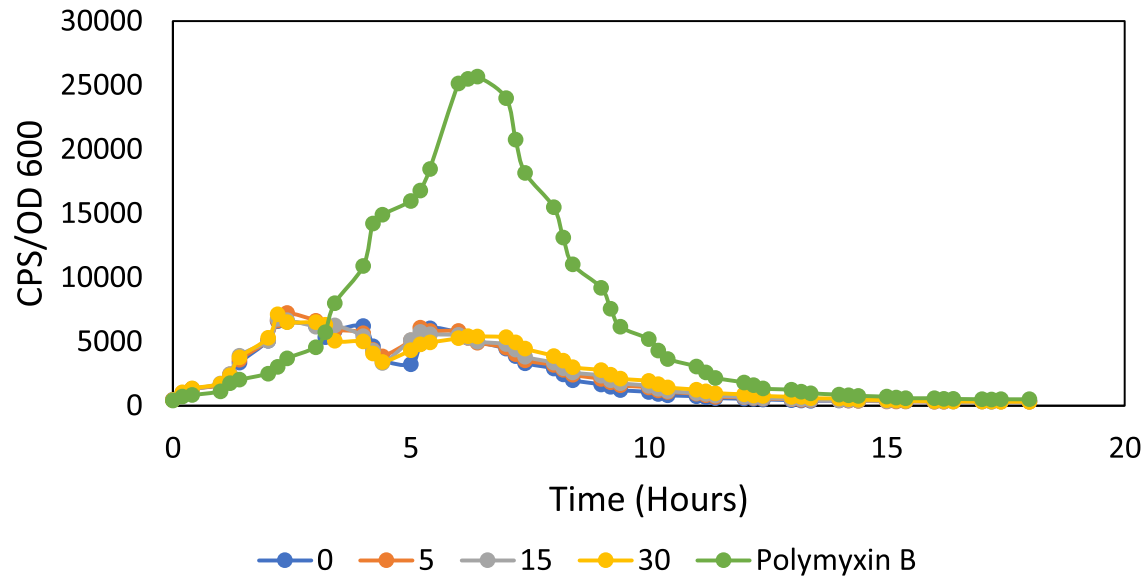

b

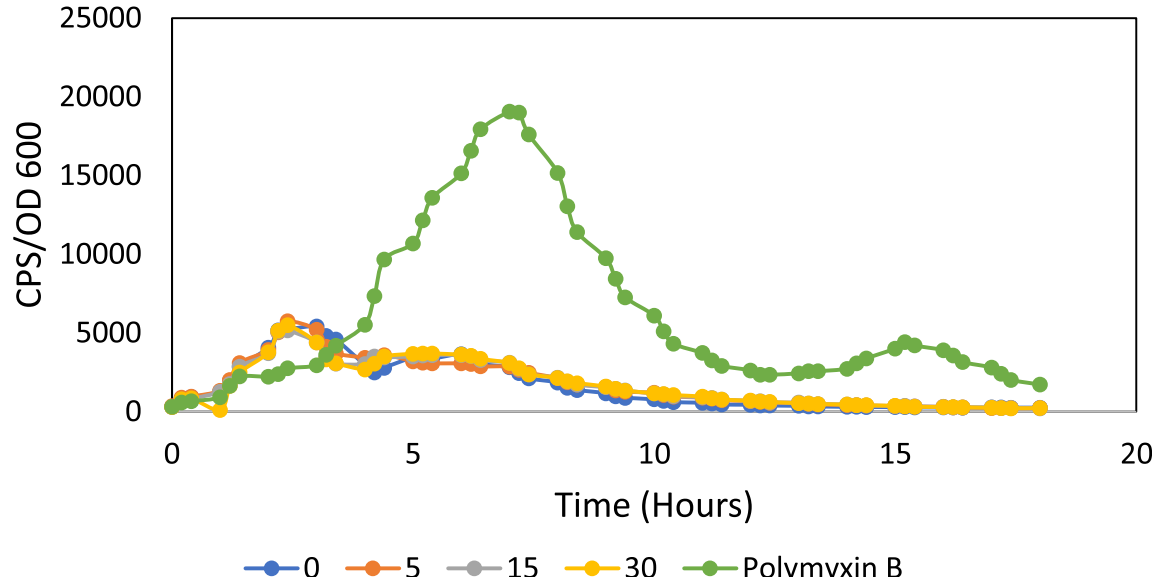

Fig. 2 So-D2 and MtDef5 $\gamma$-core motif peptides fail to induce PA4774 gene expression. Effects of So-D2 $\gamma$-core peptide (a) and MtDef5 $\gamma$-core peptide (b) at sub-minimal inhibitory concentrations of $0,5,15$, or $30 \mu \mathrm{g} / \mathrm{mL}$ or polymyxin B at $0.5 \mu \mathrm{g} / \mathrm{mL}$ on the expression of the PA4774.:/ux transcriptional fusion in planktonic cultures in LB broth. Gene expression was normalized for growth and counts per second (CPS)/OD600 values for the average of the triplicates are presented. Each growth experiment was performed three times and representative curves are shown. The standard errors were within $10 \%$ of the mean

Table 4 Membrane permeating activity of the $\gamma$-core motif defensin peptides against Pseudomonas aeruginosa strains ${ }^{a}$

\begin{tabular}{|c|c|c|c|c|}
\hline \multirow[t]{2}{*}{ Treatment } & \multicolumn{2}{|c|}{ PA3553:Iux } & \multicolumn{2}{|c|}{ PA4774:Iux } \\
\hline & $\%$ Live $^{\mathbf{b}}$ & $\%$ Dead $^{\mathbf{c}}$ & $\%$ Live & $\%$ Dead \\
\hline MtDef4 core & $71.6 \pm 5.4$ & $28.4 \pm 2.5$ & $49.4 \pm 3.8$ & $50.8 \pm 1.3$ \\
\hline MtDef5A core & $99.0 \pm 0.8$ & $0.9 \pm 0.3$ & $95.4 \pm 1.7$ & $4.6 \pm 2.1$ \\
\hline
\end{tabular}

${ }^{a}$ The mean percentage of colonies are reported \pm SE of three independent experiments $(n=3)$

bive cells have intact cell membranes and were stained fluorescent green 'Dead cells are permeable to propidium iodine and were stained fluorescent red, which indicates membrane disruption bacterial outer membrane damage following MtDef4 treatment (Table 4, Fig. S1). The PA4774::lux mutant is deficient in production of outer membrane spermidine, a polyamine, which serves as a substitute for inorganic cations that bind to and stabilize LPS in the outer membrane [36]. Antimicrobial peptides compete with cations for binding to LPS, but spermidine protects the outer membrane by ensuring that the negative surface charges are masked [25]. High concentrations of exogenous spermidine $(20 \mathrm{mM})$ have been demonstrated to increase the resistance of $P$. aeruginosa to cationic peptides [37]. Therefore, bacteria with high production levels of spermidine or other polyamines may be more resistant to plant defensin treatments. 
Table 5 BLASTn results from the Pseudomonas Genome Database identifying the transposon insertion site in the Pseudomonas syringae pv. syringae ALF3 Tn5 mutant strains

\begin{tabular}{lll}
\hline Pseudomonas syringae pv. syringae ALF3 mutant strain & Transposon insertion site & Interrupted Gene \\
\hline ALF3::Tn5-1 & 1346 in scaffold 32544_1.1 & 16S ribosomal RNA gene (RS24240) \\
ALF3::Tn5-2 & 5170 in scaffold 32544_1.1 & 23S ribosomal RNA gene (RS24220) \\
\hline
\end{tabular}

The emergence of multidrug-resistant (MDR) gramnegative bacterial isolates has led to the renewed usage of both polymyxin B and colistin (polymyxin E) as therapeutic agents [38]. Polymyxins have a polycationic ring that binds to the LPS on the outer bacterial membrane and competitively displaces $\mathrm{Ca}^{2+}$ and $\mathrm{Mg}^{2+}$ leading to membrane destabilization and increased permeability [39]. With the increased prevalence of polymyxin treatments, polymyxin-resistant $P$. aeruginosa isolates have been reported worldwide [40, 41]. Throughout our study, a polymyxin B treatment was used as positive control against $P$. aeruginosa. In all lux-reporter assays, the plant defensin treatments displayed rapid levels of lux induction, and lux-expression was induced faster with plant defensin treatments compared to polymyxin $B$ treatments (Fig. 1). These different induction dynamics in the lux-reporter assays suggest that plant defensins and polymyxin B have different MOAs on the outer membrane. Therapeutic compounds with novel MOAs are needed to treat MDR bacterial isolates, and plant defensins may be an untapped reservoir.

The transposon insertion mutants of $P$. syringae pv. syringae, ALF3::Tn5-1 and ALF3::Tn5-2, had increased resistance to MtDef4 $\gamma$-core motif peptide treatments, which may be due to amino acid synthesis mutations or changes in ribosome structure (Table 5). The ribosome is a common target for antibacterial compounds because the alteration of bacterial ribosomes causes disruption of translation [42]. For example, aminoglycoside antibiotics target 16S rRNA in the small ribosomal subunit and tylosin targets $23 \mathrm{~S}$ rRNA in the large ribosomal subunit $[43,44]$. Target site mutations are a frequently utilized bacterial resistance mechanism. To gain resistance to several antibiotics, Mycobacterium tuberculosis acquired mutations in multiple regions of the rrs gene, which encodes $16 \mathrm{~S}$ rRNA [45]. But, the multiplicity of rRNA genes in most bacterial species slows the development of this type of resistance [46]. Also, the antifungal MOAs of MtDef4 against Fusarium graminearum and Neurospora crassa requires $y$-core motif mediated entry into fungal cells, which implies that MtDef4 has an intracellular target [47].

Our results suggest that the antibacterial MOA of the MtDef4 $y$-core motif peptide may involve ribosomal targeting, and the transposon insertions in $P$. syringae pv. syringae rRNA encoding genes could be target site mutations leading to increased MtDef4 resistance. Furthermore, spermidine interacts closely with RNA because in
E. coli cells spermidine exists predominantly as a polyamine-RNA complex [48]. Polyamines play crucial roles as modulators of RNA structure and can induce changes in RNA structure in context-dependent manner [49]. Polyamine binding to $23 \mathrm{~S}$ rRNA on the central loop region of domain $\mathrm{V}$, a site where several antibiotics are known to bind, caused structural alterations, which is suggested to restrict spiramycin binding to the ribosome [50]. In addition to having decreased outer membrane spermidine content, PA4774::lux may also have a reduction of intracellular spermidine. Both spermidine and MtDef4 may normally interact with 23 and $16 \mathrm{~S}$ rRNA, but in PA4774::lux, this intracellular spermidinebased protection does not occur, which leads to increased susceptibility to MtDef4. The $P$. syringae pv. syringae transposon insertion mutants may also disrupt the interaction between MtDef4 and rRNA, which would explain the observed resistance to MtDef4. Additionally, the antibacterial MOA of MtDef4 against different Pseudomonas species may not be conserved or multiple MOAs could be utilized. The AMP melittin, the main component of European honeybee (Apis mellifera) venom, killed bacterial cells of the plant pathogen Xanthomonas oryzae pv. oryzae using multiple MOAs including membrane permeabilization, inhibition of protein synthesis, and DNA/RNA binding [51]. Also, the antifungal MOA of MtDef4 is not conserved between ascomycete fungi, N. crassa and F. graminearum [52].

\section{Conclusions}

In this report, we gain insights into the antibacterial MOA of plant defensins against two pseudomonads, $P$. aeruginosa and $P$. syringae pv. syringae. In $P$. aeruginosa, we propose that MtDef4 and So-D2 interact with the bacterial outer membrane and possibly create pores leading to bacterial cell death. MtDef5 appears to have a different antibacterial MOA where outer membrane binding is not as vital and, therefore, may have an intracellular target. This hypothesis is consistent with the reported antibacterial MOA of MtDef5 against $X$. campestris pv. campestris in which DNA binding by MtDef5 likely interferes with DNA synthesis and transcription [15]. Additionally, plant defensins seem to have a different MOA than polymyxin B. The $P$. syringae pv. syringae mutational analysis suggests that MtDef4 may also target the ribosome and interfere with bacterial translation. Resistance mechanisms that bacteria use to 
combat MtDef4 and other plant defensins may include increased cell membrane thickness through outer membrane spermidine synthesis or target site mutations. Plant defensin $\gamma$-core motif peptides can be utilized for the development of treatments against both plant and human bacterial pathogens and for furthering knowledge of mechanisms of antimicrobial resistance.

\section{Methods}

\section{Bacterial strains and growth media}

All bacterial strains utilized in this study are listed in Table 2. Pseudomonas aeruginosa strains were obtained from Dr. Lewenza at the University of Calgary. The $P$. aeruginosa lux-reporter strains have inactivated lipopolysaccharide (LPS) modification genes, which are bacterial genes involved in the resistance to cationic antimicrobial peptides. PA4774::lux has an interrupted outer membrane surface spermidine synthesis gene. PA3553:: lux has an interrupted lipid A modification gene, which is responsible for the addition of aminoarabinose to lipid A. When the lux-reporter bacteria produce bioluminescence, they act as a real-time reporter for the induction of the inactivated gene [26]. PAO1 was used as the wild type strain of $P$. aeruginosa. The $P$. aeruginosa strains were cultured on Luria-Bertani (LB) agar (Difco, Sparks, $\mathrm{MD})$ at $37^{\circ} \mathrm{C}$. From a glycerol stock, the sequenced bacterial strain, Pseudomonas syringae pv. syringae ALF3, originally isolated from an infected alfalfa plant near Cheyenne, WY, was cultured on nutrient broth yeast extract (NBY) agar at $30^{\circ} \mathrm{C}$ [28]. ALF3 was used as the wild type strain of $P$. syringae pv. syringae.

\section{Plant defensin peptide synthesis}

The $\gamma$-core motif peptides derived from plant defensins, MtDef4, MtDef5A, and So-D2 [16, 53, 54] (Table 1) were chemically synthesized and purified by HPLC (LifeTein, Somerset, NJ). Lyophilized defensin peptides were rehydrated in sterile water prior to each assay.

\section{Determination of plant defensin antibacterial activity against Pseudomonas aeruginosa}

To quantify defensin antibacterial activity, a spread-plate assay was used as previously described [3]. This assay was repeated three times for each strain of $P$. aeruginosa. Lawns of $P$. aeruginosa were grown on acidic LB $(\mathrm{pH}$ adjusted to 5.5 with $\mathrm{HCl}$ ) plates for $15 \mathrm{~h}$ at $37^{\circ} \mathrm{C}$, conditions which induce antimicrobial peptide resistance mechanisms [31]. The plates were flooded with sterile water to harvest the bacteria. Cultures were diluted with sterile water to an $\mathrm{OD}_{600}$ of 0.1 . In microcentrifuge tubes, $200 \mu \mathrm{L}$ of bacteria were incubated at $37^{\circ} \mathrm{C}$ with shaking for $3 \mathrm{~h}$ with various concentrations of a $\gamma$-core motif defensin peptide $(0,2.5,5,10,20$, or $30 \mu \mathrm{g} / \mathrm{mL})$. After the defensin peptide treatment, 10-fold serial dilutions were made, and $100 \mu \mathrm{L}$ were plated in triplicate onto LB plates. Colony forming units (CFUs) were counted for $P$. aeruginosa after incubation for $24 \mathrm{~h}$ at $37^{\circ} \mathrm{C}$. Regression of the average CFUs across experimental replications versus the defensin peptide concentration was used to create a dose response curve using Microsoft Excel 2016. From these dose response curves, the $\mathrm{IC}_{50}$ value, the amount of $\gamma$-core motif defensin peptide needed to inhibit the growth of bacterial strains by $50 \%$, was calculated. The $\mathrm{IC}_{50}$ values are presented as mean \pm standard error from the three repeated experiments.

\section{Lux-reporter gene expression assay}

Lux-reporter gene expression assays, adapted from Mulcahy et al. (2008), were performed in a high-throughput manner using 96-well microplates. P. aeruginosa cultures were grown overnight in acidic LB broth adjusted to a $\mathrm{pH}$ of 5.5. Overnight cultures were diluted by 1000 into LB broth, and $150 \mu \mathrm{L}$ of diluted culture medium with $\gamma$ core motif defensin peptide added at a sublethal concentration $(0,5,15$, or $30 \mu \mathrm{g} / \mathrm{mL})$ was added to flat clear bottom 96-well microplates (Corning, Corning, NY) and overlaid with $50 \mu \mathrm{L}$ of mineral oil to prevent evaporation. As a positive control, the antibiotic, polymyxin B, which is known to cause high gene induction of the luxreporter strains, was added at a sublethal concentration of $0.5 \mu \mathrm{g} / \mathrm{mL}$. Samples were assayed in triplicate. Microplate cultures were incubated at $37^{\circ} \mathrm{C}$ for $18 \mathrm{~h}$ in a Synergy H1 microplate reader (BioTek, Winooski, VT) with optical density $(600 \mathrm{~nm})$ and luminescence (counts per second [CPS]) readings taken every $20 \mathrm{~min}$ throughout the incubation period. Gene expression values were normalized to growth $\left(\mathrm{CPS} / \mathrm{OD}_{600}\right)$.

\section{Assessment of bacterial membrane permeability through fluorescent microscopy}

The PA4774::lux and PA3553::lux strains of $P$. aeruginosa were grown overnight in acidic LB broth adjusted to a $\mathrm{pH}$ of 5.5. Overnight cultures were diluted by 1000 in sterile water. In microcentrifuge tubes, $150 \mu \mathrm{L}$ of the diluted bacterial suspension was treated with $30 \mu \mathrm{g} / \mathrm{mL}$ of either the MtDef4 or MtDef5 $\gamma$-core peptide and incubated at $37^{\circ} \mathrm{C}$ for $3 \mathrm{~h}$ with shaking. Defensin treated bacteria were stained using a LIVE/DEAD BacLight Bacterial Viability Kit (Thermo Fisher) following the manufacturer's instructions. On a slide with one droplet of BacLight mounting oil, $5 \mu \mathrm{L}$ of the stained bacterial suspension was observed using fluorescent microscopy.

\section{Pseudomonas syringae pv. syringae transposon mutagenesis}

An EZ-Tn5 < R6Kyori/KAN-2 > Tnp Transposome Kit (Lucigen, Middleton, WI) was used to generate mutants of Pseudomonas syringae pv. syringae strain ALF3 
through random transposon insertions. The transposome was transformed into the ALF3 strain using the $P$. syringae pv. syringae electroporation protocol previously described by Scholz-Schroeder [55]. The transformed bacteria were plated onto NBY agar plates with $50 \mathrm{mg} / \mathrm{L}$ kanamycin and incubated at $25^{\circ} \mathrm{C}$ for $48 \mathrm{~h}$. Colonies were pooled by flooding the plates with sterile water. Bacterial cultures were diluted with sterile water to an $\mathrm{OD}_{600}$ of 0.1. In microcentrifuge tubes, the MtDef4 $\gamma$ core motif peptide at $80 \mu \mathrm{g} / \mathrm{mL}$, approximately 10 times the $\mathrm{IC}_{50}$ concentration, was added to $200 \mu \mathrm{L}$ of the transformed bacteria, and the cultures were incubated at $25^{\circ} \mathrm{C}$ with shaking for $3 \mathrm{~h}$. After the defensin treatment, 10 -fold serial dilutions were made and $100 \mu \mathrm{L}$ were plated in triplicate onto NBY plus kanamycin plates. Plates were grown at $25^{\circ} \mathrm{C}$ overnight. Single colonies were selected, restreaked on NBY plus kanamycin plates, grown overnight at $25^{\circ} \mathrm{C}$, and the defensin treatment was repeated. From the recovered $P$. syringae pv. syringae mutants resistant to the MtDef4 $\gamma$-core motif peptide, genomic DNA was extracted and digested with EcoRI (NEB, Ipswich, MA). The DNA was self-ligated using T4 DNA ligase (NEB). Electrocompetent TransforMax EC100D pir-116 E. coli (Lucigen) were electroporated with $2 \mu \mathrm{L}$ of the ligation mix. The transformed E. coli were plated on LB agar plus $50 \mathrm{mg} / \mathrm{L}$ kanamycin and grown overnight at $37^{\circ} \mathrm{C}$. Plasmid DNA was extracted using a QIAprep Spin Miniprep Kit (Qiagen, Valencia, CA). The plasmid DNA was Sanger sequenced on both sides of the transposon insertion at the University of Minnesota Genomics Center using the supplied primers from the Tnp Transposome kit, KAN-2 FP-1 (5'-ACCTACAACAAAGCTCTCATCAACC - 3') and R6KAN-2 RP-1 (5' - CTACCCTGTGGAACACCTACAT CT-3'). The resulting DNA sequences near the transposon insertion were validated using Sequencer (Gene Codes Corporation, Ann Arbor, MI). Nucleotide BLAST searches using the Pseudomonas Genome Database [56] were performed on the DNA sequences near the transposon insertion site to identify the locations in the ALF3 genome of the insertions and the corresponding interrupted genes with annotations.

\section{Supplementary information}

Supplementary information accompanies this paper at https://doi.org/10. 1186/s12866-020-01852-1.

\footnotetext{
Additional file 1: Figure S1. MtDef4 $\gamma$-core motif peptide causes membrane permeabilization in both the PA4774:.:/ux (a) and PA3553:.//ux (b) strains of Pseudomonas aeruginosa. Observed fluorescence using optical filters set for SYTO 9 green-fluorescent staining (left), propidium iodine red-fluorescent staining (center), and the merged images (right). Green fluorescence reveals bacterial cells with intact membranes while red fluorescence reveals bacterial cells with damaged membranes. Scale bar is $5 \mu \mathrm{m}$.

Additional file 2: Figure S2. MtDef5 $\gamma$-core motif peptide causes limited membrane permeabilization in both the PA4774.:/Ux (a) and
}

PA3553::./ux (b) strains of Pseudomonas aeruginosa. Observed fluorescence using optical filters set for SYTO 9 green-fluorescent staining (left), propidium iodine red-fluorescent staining (center), and the merged images (right). Green fluorescence reveals bacterial cells with intact membranes while red fluorescence reveals bacterial cells with damaged membranes. Scale bar is $5 \mu \mathrm{m}$.

\section{Abbreviations}

AMP: Antimicrobial peptide; MsDef1: Medicago sativa defensin 1;

NaD1: Nicotiana alata defensin 1; So-D2: Spinacia oleracea defensin 2; J11: Capsicum annum defensin 1; MIC: Minimum inhibitory concentration; PaDef: Persea americana var. drymifolia defensin; MOA: Mode of action; MtDef5: Medicago truncatula defensin $5 ; I_{50}$ : Inhibitory concentration 50\%; LPS: Lipopolysaccharide; HNP-1: Human a-defensin; HBD3: Human $\beta$ defensin-3; MtDef4: Medicago truncatula defensin 4; MDR: Multidrug-resistant; LB: Luria-Bertani; NBY: Nutrient broth yeast extract; CFU: Colony forming units; CPS: Counts per second

\section{Acknowledgements}

Not applicable.

\section{Authors' contributions}

AES, SL, and DAS conceived and the designed the research. AES conducted the experiments. AES, SL, and DAS wrote the manuscript. All authors read and approved the manuscript.

\section{Funding}

The work was supported by funding by the USDA-ARS project 5062-12210003D and the Torske Klubben Fellowship. The funding bodies had no role in the design of the study, collection, analysis, or interpretation of data, or writing of the manuscript.

\section{Availability of data and materials}

The raw data generated and analyzed during this study are available from the corresponding author on reasonable request.

Ethics approval and consent to participate

Not applicable.

Consent for publication

Not applicable.

\section{Competing interests}

The authors declare that they have no competing interests.

\section{Author details}

'Department of Plant Pathology, 1991 Upper Buford Circle, University of Minnesota, St. Paul, MN 55108, USA. ²Department of Biology, Dakota State University, 820 N Washington Ave, Madison, SD 57042, USA. ${ }^{3}$ Department of Microbiology and Infectious Disease, 3330 Hospital Dr. N.W., University of Calgary, Calgary, AB T2N 4Z6, Canada. ${ }^{4}$ Faculty of Science and Technology, 1 University Dr., Athabasca University, Athabasca, AB T9S 3A3, Canada.

${ }^{5}$ USDA-ARS, Plant Science Research Unit, 1991 Upper Buford Circle, St. Paul, MN 55108, USA.

Received: 6 January 2020 Accepted: 12 June 2020

Published online: 19 June 2020

References

1. Goyal RK, Mattoo AK. Multitasking antimicrobial peptides in plant development and host defense against biotic/abiotic stress. Plant Sci. 2014; 228:135-49.

2. Tam JP, Wang S, Wong KH, Tan WL. Antimicrobial peptides from plants. Pharmaceuticals. 2015:8:711-57.

3. Sathoff AE, Velivelli S, Shah DM, Samac DA. Plant defensin peptides have antifungal and antibacterial activity against human and plant pathogens. Phytopathology. 2019;109:402-8.

4. Franco OL. Peptide promiscuity: an evolutionary concept for plant defense. FEBS Lett. 2011;585:995-1000. 
5. van der Weerden NL, Anderson MA. Plant defensins: common fold, multiple functions. Fungal Biol Rev. 2013;26:121-31.

6. Gao AG, Hakimi SM, Mittanck CA, Wu Y, Woerner BM, Stark DM, Shah DM, Liang J, Rommens CMT. Fungal pathogen protection in potato by expression of a plant defensin peptide. Nat Biotechnol. 2000;18:1307-10.

7. Gaspar YM, McKenna JA, McGinness BS, Hinch J, Poon S, Connelly AA, Anderson MA, Heath RL. Field resistance to Fusarium oxysporum and Verticillium dahliae in transgenic cotton expressing the plant defensin NaD1. J Exp Bot. 2014;65:1541-50.

8. Sathoff AE, Samac DA. Antibacterial activity of plant defensins. Mol PlantMicrobe Interact. 2019;32:507-14.

9. Mirkov TE, Gonzalez-Ramos J. Pathogen resistant citrus compositions, organisms, systems, and methods. U.S. Patent application. 2014; No.14/ 139,791 .

10. Guillén-Chable F, Arenas-Sosa I, Islas-Flores I, Corzo G, Martinez-Liu C, Estrada G. Antibacterial activity and phospholipid recognition of the recombinant defensin J1-1 from Capsicum genus. Protein Expr Purif. 2017;136:45-51.

11. Guzmán-Rodríguez JJ, López-Gómez R, Suárez-Rodríguez LM, SalgadoGarciglia R, Rodríguez-Zapata LC, Ochoa-Zarzosa A, López-Meza JE. Antibacterial activity of defensin PaDef from avocado fruit (Persea americana var. drymifolia) expressed in endothelial cells against Escherichia coli and Staphylococcus aureus. Biomed Res Int. 2013;2013:986273.

12. Cools T, Struyfs C, Cammue B, Thevissen K. Antifungal plant defensins: increased insight in their mode of action as a basis for their use to combat fungal infections. Future Microbiol. 2017;12:441-54.

13. Lacerda AF, Vasconcelos ÉAR, Pelegrini PB. Grossi de Sa MF. Antifungal defensins and their role in plant defense. Front Microbiol. 2014;5:1-10.

14. Parisi K, Shafee TMA, Quimbar P, van der Weerden NL, Bleackley MR, Anderson MA. The evolution, function and mechanisms of action for plant defensins. Semin Cell Dev Biol. 2019;88:107-18.

15. Velivelli SLS, Islam KT, Hobson E, Shah DM. Modes of action of a bi-domain plant defensin MtDef5 against a bacterial pathogen Xanthomonas campestris. Front Microbiol. 2018;9:934.

16. Islam KT, Velivelli SLS, Berg RH, Oakley B, Shah DM. A novel bi-domain plant defensin MtDef5 with potent broad-spectrum antifungal activity binds to multiple phospholipids and forms oligomers. Sci Rep. 2017;7:16157.

17. Ageitos JM, Sánchez-Pérez A, Calo-Mata P, Villa TG. Antimicrobial peptides (AMPs): ancient compounds that represent novel weapons in the fight against bacteria. Biochem Pharmacol. 2017;133:117-38.

18. Guilhelmelli F, Vilela N, Albuquerque P, Derengowski L d S, Silva-Pereira I, Kyaw CM. Antibiotic development challenges: The various mechanisms of action of antimicrobial peptides and of bacterial resistance. Front Microbiol. 2013:4:353.

19. Morgera F, Antcheva N, Pacor S, Quaroni L, Berti F, Vaccari L, Tossi A. Structuring and interactions of human $\beta$-defensins 2 and 3 with model membranes. J Pept Sci. 2008;14:518-23.

20. Zhang $Y, L u$ W, Hong $M$. The membrane-bound structure and topology of a human a-defensin indicate a dimer pore mechanism for membrane disruption. Biochemistry. 2010;49:9770-82.

21. Sass V, Pag U, Tossi A, Bierbaum G, Sahl HG. Mode of action of human $\beta$ defensin 3 against Staphylococcus aureus and transcriptional analysis of responses to defensin challenge. Int Med Microbiol. 2008;298:619-33.

22. Anaya-López JL, López-Meza JE, Ochoa-Zarzosa A. Bacterial resistance to cationic antimicrobial peptides. Crit Rev Microbiol. 2013;39:180-95.

23. McPhee JB, Lewenza S, Hancock REW. Cationic antimicrobial peptides activate a two-component regulatory system, PmrA-PmrB, that regulates resistance to polymyxin $B$ and cationic antimicrobial peptides in Pseudomonas aeruginosa. Mol Microbiol. 2003;50:205-17.

24. Mulcahy H, Charron-Mazenod L, Lewenza S. Extracellular DNA chelates cations and induces antibiotic resistance in Pseudomonas aeruginosa biofilms. PLoS Pathog. 2008;4:e1000213.

25. Johnson L, Mulcahy H, Kanevets U, Shi Y, Lewenza S. Surface-localized spermidine protects the Pseudomonas aeruginosa: outer membrane from antibiotic treatment and oxidative stress. J Bacteriol. 2012;194:813-26.

26. Lewenza S, Falsafi RK, Winsor G, Gooderham WJ, Mcphee JB, Brinkman FSL, Hancock REW. Construction of a mini-Tn5-luxCDABE mutant library in Pseudomonas aeruginosa PAO1: a tool for identifying differentially regulated genes. Genome Res. 2005;15:583-9.

27. Nemchinov LG, Shao J, Lee MN, Postnikova OA, Samac DA. Resistant and susceptible responses in alfalfa (Medicago sativa) to bacterial stem blight caused by Pseudomonas syringae pv. syringae. PLoS One. 2017;12:1-20.
28. Harrison J, Dornbusch MR, Samac D, Studholme DJ. Draft genome sequence of Pseudomonas syringae pv. Syringae ALF3 isolated from alfalfa. Genome Announc. 2016;4:e01722-15.

29. Stover CK, Pham XQ, Erwin AL, Mizoguchi SD, Warrener P, Hickey MJ, Brinkman FS, Hufnagle WO, Kowalik DJ, Lagrou M, Garber RL, Goltry L, Tolentino E, Westbrock-Wadman S, Yuan Y, Brody LL, Coulter SN, Folger KR, Kas A, Larbig K, Lim R, Smith K, Spencer D, Wong GK, Wu Z, Paulsen IT, Reizer J, Saier MH, Hancock RE, Lory S, Olson MV. Complete genome sequence of Pseudomonas aeruginosa PAO1, an opportunistic pathogen. Nature. 2000;406:959-64.

30. Lewenza S. Extracellular DNA-induced antimicrobial peptide resistance mechanisms in Pseudomonas aeruginosa. Front Microbiol. 2013;4:1-6.

31. Wilton M, Charron-Mazenod L, Moore R, Lewenza S. Extracellular DNA acidifies biofilms and induces aminoglycoside resistance in Pseudomonas aeruginosa. Antimicrob Agents Chemother. 2016;60:544-53.

32. Berney M, Hammes F, Bosshard F, Weilenmann HU, Egli T. Assessment and interpretation of bacterial viability by using the LIVE/DEAD BacLight kit in combination with flow cytometry. Appl Environ Microbiol. 2007;73:3283-90.

33. Hilpert K, Volkmer-Engert R, Walter T, Hancock REW. High-throughput generation of small antibacterial peptides with improved activity. Nat Biotechnol. 2005;23:1008-12.

34. Hancock REW. Alterations in structure of the cell envelope. Ann Rev Microbiol. 1984;38:237-64.

35. Moskowitz SM, Ernst RK, Miller SI. PmrAB, a two-component regulatory system of Pseudomonas aeruginosa that modulates resistance to cationic antimicrobial peptides and addition of aminoarabinose to lipid a. J Bacteriol. 2004;186:575-9.

36. Hancock REW, Wong PGW. Compounds which increase the permeability of the Pseudomonas aeruginosa outer membrane. Antimicrob Agents Chemother. 1984;26:48-52

37. Kwon DH, Lu C. Polyamines induce resistance to cationic peptide, aminoglycoside, and quinolone antibiotics in Pseudomonas aeruginosa PAO1. Antimicrob Agents Chemother. 2006;50:1615-22.

38. Nation RL, Li J. Colistin in the $21^{\text {st }}$ century. Curr Opin Infect Dis. 2009;22: 535-43.

39. Zavascki AP, Goldani LZ, Li J, Nation RL. Polymyxin B for the treatment of multidrug-resistant pathogens: a critical review. J Antimicrob Chemother. 2007;60:1206-15.

40. Lee JY, Song JH, Ko KS. Identification of nonclonal Pseudomonas aeruginosa isolates with reduced colistin susceptibility in Korea. Microb Drug Resist. 2011;17:299-304.

41. Wang CY, Jerng JS, Cheng KY, Lee LN, Yu CJ, Hsueh PR, Yang PC. Pandrugresistant Pseudomonas aeruginosa among hospitalized patients: clinical features, risk-factors and outcomes. Clin Microbiol Infect. 2006;12:63-8.

42. Tenson T, Mankin A. Antibiotics and the ribosome. Mol Microbiol. 2006;59: 1664-77.

43. Hansen JL, Ippolito JA, Ban N, Nissen P, Moore PB, Steitz TA. The structures of four macrolide antibiotics bound to the large ribosomal subunit. Mol Cell. 2002;10:117-28.

44. Ogle JM, Brodersen DE, Clemons WM Jr, Tarry MJ, Carter AP, Ramakrishnan $V$. Recognition of cognate transfer RNA by the 30 S ribosomal subunit. Science. 2001;292:897-902.

45. Nguyen $\mathrm{QH}$, Contamin L, Nguyen TVA, Bañuls AL. Insights into the processes that drive the evolution of drug resistance in Mycobacterium tuberculosis. Evol Appl. 2018;11:1498-511.

46. Cundliffe E. Recognition sites for antibiotics within rRNA. In: Hill WE, Dalberg A, Garrett RA, Moore PB, Schlessinger D, Warner JR, editors. The ribosome: structure, function and evolution. Washington: American Society for Microbiology; 1990. p. 479-90.

47. Sagaram US, El-Mounadi K, Buchko GW, Berg HR, Kaur J, Pandurangi RS, Smith TJ, Shah DM. Structural and functional studies of a phosphatidic acidbinding antifungal plant defensin MtDef4: identification of an RGFRRR motif governing fungal cell entry. PLoS One. 2013;8:1-22.

48. Igarashi K, Kashiwagi K. Modulation of cellular function by polyamines. Int J Biochem Cell Biol. 2010;42:39-51.

49. Lightfoot HL, Hall J. Endogenous polyamine function - the RNA perspective. Nucleic Acids Res. 2014;42:11275-90.

50. Petropoulos AD, Xaplanteri MA, Dinos GP, Wilson DN, Kalpaxis DL. Polyamines affect diversely the antibiotic potency: insight gained from kinetic studies of the blasticidin S and spiramycin interactions with functional ribosomes. J Biol Chem. 2004;279:26518-25. 
51. Shi W, Li C, Li M, Zong X, Han D, Chen Y. Antimicrobial peptide melittin against Xanthomonas oryzae pv. oryzae, the bacterial leaf blight pathogen in rice. Appl Microbiol Biotechnol. 2016;100:5059-67.

52. El-Mounadi K, Islam KT, Hernández-Ortiz P, Read ND, Shah DM. Antifungal mechanisms of a plant defensin MtDef4 are not conserved between the ascomycete fungi Neurospora crassa and Fusarium graminearum. Mol Microbiol. 2016;100:542-59.

53. Sagaram US, Pandurangi R, Kaur J, Smith TJ, Shah DM. Structure-activity determinants in antifungal plant defensins MsDef1 and MtDef4 with different modes of action against Fusarium graminearum. PLoS One. 2011;6: e18550.

54. Segura A, Moreno M, Molina A, García-Olmedo F. Novel defensin subfamily from spinach (Spinacia oleracea). FEBS Lett. 1998;435:159-62.

55. Scholz-Schroeder BK. Electroporation and marker exchange mutagenesis of Pseudomonas syringae pv. syringae. The Plant Health Instructor. 2001. https://www.apsnet.org/edcenter/disimpactmngmnt/labexercises/ Electroporation/Pages/default.aspx Accessed 28 Jan 2018.

56. Winsor GL, Griffiths EJ, Lo R, Dhillon BK, Shay JA, Brinkman FSL. Enhanced annotations and features for comparing thousands of Pseudomonas genomes in the Pseudomonas genome database. Nucleic Acids Res. 2016; 44:D646-53.

\section{Publisher's Note}

Springer Nature remains neutral with regard to jurisdictional claims in published maps and institutional affiliations.

Ready to submit your research? Choose BMC and benefit from:

- fast, convenient online submission

- thorough peer review by experienced researchers in your field

- rapid publication on acceptance

- support for research data, including large and complex data types

- gold Open Access which fosters wider collaboration and increased citations

- maximum visibility for your research: over $100 \mathrm{M}$ website views per year

At $\mathrm{BMC}$, research is always in progress.

Learn more biomedcentral.com/submissions 